MATTEO NACCI

Pontificia Università Lateranense

\title{
CONSIDERAZIONI STORICO-GIURIDICHE SULLE 'RELAZIONI CHIESA-MONDO' NEL CONCILIO DI TRENTO E NEL CONCILIO VATICANO II
}

Sommario: Introduzione. - 1. Il Concilio di Trento e le 'relazioni Chiesa-Mondo'. - 2. Il Concilio Vaticano II e le 'relazioni Chiesa-Mondo'. - 2.1. La Costituzione dogmatica sulla Chiesa Lumen Gentium. - 2.2. La Costituzione pastorale sulla Chiesa nel mondo contemporaneo Gaudium et Spes. - 2.3. La Dichiarazione sulla libertà religiosa Dignitatis Humanae.

\section{Introduzione}

La circostanza che ha determinato la stesura del presente contributo $^{1} \mathrm{mi}$ ha permesso di sintetizzare un periodo storico lungo più di quattro secoli - dal Concilio di Trento (1545-1563) al Concilio Vaticano II (1962-1965) - e al tempo stesso rilevantissimo per la scienza giuridica denominata 'diritto pubblico ecclesiastico' che fin dal suo sorgere si è occupata, dal punto di vista teorico, della "regolamentazione" dei rapporti fra la Chiesa e gli ordinamenti civili. Nell'affrontare ormai da diversi anni lo studio di questa peculiare

\footnotetext{
${ }^{1}$ Conferenza tenuta presso l'Universidad Católica de Murcia (Spagna) in occasione della I Jornadas anuales de Derecho y Teología, Derecho y Crisis. Consecuencias canónicas de los tiempos de cambio (21 maggio 2015). Il presente contributo, con alcune modifiche, è destinato anche alla pubblicazione nella rivista scientifica Vergentis della Cattedra Internazionale di Ricerca 'Innocenzo III'.
} 
branca del diritto canonico, recentemente racchiuso in una pubblicazione monografica ${ }^{2}$, sono giunto alla precisa consapevolezza che il diritto pubblico ecclesiastico ha un grande valore culturale e che è utile e necessario, da storico del diritto, scavare a fondo per comprenderne bene le varie tappe storiche e le rilevantissime implicazioni contemporanee.

\section{Il Concilio di Trento e le 'relazioni Chiesa-Mondo'}

All'interno dei documenti del Concilio di Trento (1545-1563), risposta immediata della Chiesa al dilagare della dottrina protestante $^{3}$, appare chiaro, oltre ad una reazione del mondo cattolico alla "tempesta" provocata dal monaco di Eisleben ${ }^{4}$, lo sforzo di presen-

${ }^{2}$ M. NACCI, Chiesa e Stato dalla potestà contesa alla sana cooperatio. Un profilo storico-giuridico, Lateran University Press, Roma 2015.

${ }^{3}$ Riguardo alla Riforma protestante si veda L. von Ranke, Deutsche Geschichte im Zeitalter der Reformation, 6 voll., Duncker und Humblot, Berlin 1839-1847; F. von Bezold, Storia della Riforma in Germania, Stab. Tip. Della Società Editrice Libraria, Milano 1900; E. GoTHEIN, Schriften zur Kulturgeschichte der Renaissance, Reformation und Gegenreformation, II, Reformation und Gegenreformation, Duncker und Humblot, München 1924; P. Imbart De La Tour, Origines de la Réforme, II, L'Église catholique: la crise et la renaissance, Librarie D’Argences, Melun 1946; E. G. LÉOnARD, Histoire générale du Protestantisme, 3 voll., Presses universitaires de France, Paris 1961-1964; P. Tillich, L'era protestante, Claudiana, Torino 1972; K. Aland, Geschichte der Christenheit, II voll., Gütersloher Verl.-Haus Mohn, Gütersloh 1980-1982; S. H. HENDrix, Tradition and authority in the Reformation, Ashgate variorum, Aldershot 1996; F. MussgnUG, Lutero e la riforma protestante, Giunti, Firenze 2003; D. K. McKıм, The Cambridge Companion to Martin Luther, Cambridge University Press, Cambridge 2003; R. H. BAInton, Lutero e la riforma protestante, Mondadori, Milano 2011.

${ }^{4}$ Sulla vita, le opere e il pensiero di Martino Lutero si veda H. Denifle, Luther und Luthertum in der ersten Entwickelung, Verlag von Franz Kirchheim, Mainz 1904-1909; E. Hirsch, Lutherstudien, C. Bertelsmann, Gütersloh 1954; O. ClemeN (ed.), Luthers Werke in Auswahl, De Gruyter, Berlin 1933-1962; R. H. BAinton, Lutero, Einaudi, Torino 1966; G. Zschabitz, Martin Luther, VEB deutscher Verlag der Wissenschaften, Berlin 1967; T. HARnack, Luthers Theologie mit besonderer Beziehung auf seine Versöhnungs und Erlösunggslehere, Ed. RODOPI, Amsterdam 1969; G. Ebeling, Lutherstudien, Mohor, Tübingen 1971; M. Miegge, Lutero giovane, Feltrinelli, Milano 1977; K. Bornкamm - G. Ebeling (edd.), Ausgewählte 
tare in forma positiva la dottrina della Chiesa Cattolica ${ }^{5}$. Anche se quest'ultima non si è "definita” ufficialmente durante l'Assise tridentina, le successive speculazioni dei teologi cattolici sulle "questioni definitorie" della Chiesa costituiranno la base delle teorie giuridiche dei giuspubblicisti di Würzburg che opposero, con tono apologetico, agli errores protestantium ${ }^{6}$.

Secondo quanto rileva Willeart in uno studio sulla Restaurazione cattolica, era necessario, soprattutto dopo il Concilio di Trento, definire con chiarezza i titoli della Chiesa, messi in discussione da Lutero, per permetterle di trasmettere con certezza l'oggetto della

Schriften, Insel, Frankfurt-am-Main 1982; V. Press - D. Stievermann (edd.), Martin Luther, Klett-Cotta, Stuttgart 1986; L. Febvre, Martin Lutero, Laterza, Roma 1996; R. Coggi, Ripensando Lutero, ESD, Bologna 2004; O. H. Pesc, Martin Lutero. Introduzione storica e teologica, Queriniana, Brescia 2007; D. KAMPEN, Introduzione alla teologia luterana, Claudiana, Torino 2011.

${ }^{5}$ Per comprendere correttamente le fasi genetiche del Concilio di Trento in relazione alla posizione di Lutero e dei protestanti si veda, A. Fliche - V. MARTIN (edd.), Storia della Chiesa, XVII, La Chiesa all'epoca del Concilio di Trento (15451563), di L. Cristiani, SAIE, Torino 1981, pp. 325-336. Per ulteriori studi sull'Assise conciliare e il diritto tridentino si veda H. Jedin, Geschichte des Konzils von Trient, 5 voll., Herder, Freiburg 1957-1977; G. Alberigo (ed.), Conciliorum Oecumenicorum Decreta, Istituto per le Scienze Religiose, Bologna 1973, pp. 657-799; G. LE BRAs J. GAudemet (ed.), Histoire du droit et des institutions de l'Église en Occident, XIV, L'époque de la Réforme et du Concile de Trente, di J. Bernhard-Ch. Lefebvre-F. Rapp, Sirey, Paris 1989; G. Martina, La Chiesa nell'età dell'assolutismo, del liberalismo, del totalitarismo, I, L'età della riforma, Morcelliana, Brescia 1990, pp. 173-200; M. Venard, Il Concilio Lateranense V e il Tridentino, in: G. Alberigo (ed.), Storia dei Concili Ecumenici, Queriniana, Brescia 1990, pp. 321-368; J. GAUdEMET, Storia del diritto canonico. Ecclesia et Civitas, San Paolo, Cinisello Balsamo 1998, pp. 711-716; A. Prosperi, Il Concilio di Trento: una introduzione storica, G. Einaudi, Torino 2001; P. Prodi, Il paradigma tridentino: un'epoca della storia della Chiesa, Morcelliana, Brescia 2010; J. W. O’Malley, Trento: il racconto del Concilio, traduzione di Stefano Galli, V\&P, Milano 2013.

${ }^{6}$ Il Willeart, infatti, attesta come "fatto curioso" che «nel sec. XVI, come anche nei secoli precedenti, la Chiesa non si è definita ufficialmente, neppure al Concilio di Trento» (A. Fliche - V. Martin (edd.), Storia della Chiesa, XVIII/1, La Restaurazione cattolica dopo il Concilio di Trento (1563-1648), di L. Willeart, SAIE, Torino 1966, p. 396). 
fede $^{7}$. Nessuno, infatti, metteva in discussione la volontà fondazionale di Cristo nei confronti della Sua Chiesa, né la natura divina delle Sue parole, portatrici di salvezza eterna, per le quali era necessaria un'assoluta garanzia di infallibilità. Ciò che divise i cattolici dai protestanti fu la diversa configurazione del "mezzo" vòlto a interiorizzare il messaggio divino: l'autorità istituzionale ecclesiastica per i primi, sola Scriptura per i secondi ${ }^{8}$.

Attaccati dai protestanti, i teologi cattolici, nel periodo successivo al Concilio di Trento, si occuparono non solo di confermare la necessità dell'autorità ecclesiastica per comprendere il messaggio divino, ma anche di specificare quali fossero le autorità - Papato e gerarchia ecclesiastica - deputate a esplicitare tale infallibilità. La preoccupazione dei teologi nel periodo postridentino è rafforzare la parte istituzionale della Chiesa, il concetto di "Chiesa-istituzione" inteso come la parte visibile, organizzativa, istituzionale e terrena della stessa ${ }^{9}$. Come mai viene compiuta questa "attività di rafforzamento"? Almeno per due ordini di ragioni. Innanzitutto la Chiesa del sedicesimo secolo è ormai ben lontana dall'essere la protagonista indiscussa di una società medievale in cui il potere politico si presentava come incompiuto e il legislatore era quasi del tutto assente. "Incompiutezza del potere politico" e "appartatezza del legislatore" sono due espressioni che rendono perfettamente l'idea del "vuoto politico" in cui vive il mondo medievale e del ruolo di supplenza svolto egregiamente dalla Chiesa: di fronte ad un prìncipe occupato quasi esclusivamente a difendere i confini dei suoi domìni e a riscuotere tasse, essa mette a disposizione la sua esperienza giuridica e umana per la società medievale ${ }^{10}$.

In secondo luogo, nel Cinquecento cambia completamente la percezione filosofico-antropologica dell'essere umano. Da una concezione comunitaria del soggetto si passa ad una concezione individualista;

\footnotetext{
${ }^{7}$ A. Fliche - V. Martin (edd.), Storia della Chiesa, XVIII/1, p. 390.

${ }^{8}$ Ivi, p. 391.

${ }^{9}$ Ivi, p. 393.

${ }^{10}$ P. Grossi, L'Europa del diritto, 7 ed., Laterza, Bari 2011, pp. 11-16; 27-29.
} 
il singolo non è più soltanto una "singola individualità" che volontariamente decide di inserirsi all'interno di una communitas, ma viene considerato tale in quanto individuo capace di proiettare nel mondo esterno la sua potestas dominativa. In questo modo il singolo, grazie anche alle correnti umanistiche, non ha più bisogno di un tessuto comunitario sovraordinato (la gerarchia ecclesiastica) ma può "interagire" direttamente con Dio attraverso lo studio della Sacra Scrittura e il contatto diretto con le fonti della Rivelazione.

Come definire, allora, la Chiesa attaccata da Lutero e dai riformatori? Nonostante necessitasse maggiore protezione la parte istituzionale della Chiesa, i teologi - sulla base di frammenti scritturistici quali At 9,4; 1 Cor 12,12; Ef 1,23 - puntarono all'affermazione della sua natura invisibile, identificandola come Corpo mistico di Cristo: secondo questa teoria, infatti, «il Cristo non è soltanto il capo mistico (sacro) della Chiesa, né solamente il suo sposo; è Lui che le conferisce la sua personalità: è il suo «ministro», e forma con essa una sola persona» ${ }^{11}$. Com'è naturale, l'immagine della Chiesa come Signore Gesù Cristo non fu prerogativa dei soli cattolici ma, a seconda delle diverse peculiarità, venne utilizzata da ogni confessione ${ }^{12}$. Se protestanti e teologi cattolici erano d'accordo sull'immagine della Chiesa "invisibile", allo stesso modo si posero su fronti contrapposti sulla parte "visibile" della stessa affaticandosi ciascuno a dimostrare, secondo la celeberrima teoria delle note della Chiesa (unità, santità, apostolicità, cattolicità), le proprie attribuzioni e prerogative ${ }^{13}$.

I padri conciliari non trattarono direttamente neppure il tema delle relazioni della Chiesa con lo Stato, lasciandolo alla diplomatica gestione della Santa Sede e alla riflessione del pensiero cattolico successivo al Concilio di Trento ma indirizzandone, anche se "idealmente", la determinazione. Relazioni che, di fatto, si presentarono molto complesse e delicate nell'epoca postridentina - dalla fine del Concilio (1563) ai Trattati di Vestfalia (1648) - per una serie di fattori

\footnotetext{
${ }^{11}$ Cfr. A. Fliche - V. Martin (edd.), Storia della Chiesa, XVIII/1, p. 397.

${ }^{12}$ Ibidem.

${ }^{13}$ Ivi, pp. 399-405.
} 
rilevanti che in questa sede merita accennare. Innanzitutto il Rinascimento, il grande "termometro" che segnò il passaggio dalla mentalità medievale a quella moderna e che con i suoi elementi caratteristici contribuì a ravvivare l'ideologia protestante. Basti pensare al già accennato individualismo, tanto sociologico quanto antropologico, che condusse anche a un individualismo religioso raffigurante l'uomo fautore della propria vita religiosa e, soprattutto, extra Ecclesiam.

In secondo luogo, l'individualismo antropologico e religioso condusse irrimediabilmente verso la valorizzazione del nazionalismo. Si abbandonò definitivamente, cioè, la concezione comunitaria della società per giungere ad una visione nazionalista; il gruppo sociale era tale perché appartenente ad un contesto geografico ben determinato e, come conseguenza, si affermò il principio territoriale cuius regio eius et religio, tappa fondamentale per giungere al concetto di "religione di Stato" ${ }^{14}$. In questo panorama, da una parte lo Stato - forte dell'appoggio delle nuove fedi riformate - cercò di assorbire e trasferire ogni aspetto della vita dell'individuo, compreso quello religioso, all'interno del suo territorio; dall'altra la Chiesa, che assisteva alla costante erosione della sua competenza universale, cercò di affermare l'autorità suprema del Papato - teoria che si ripeterà costantemente nella scienza giuspubblicistica - identificandosi sempre di più con il capo visibile ${ }^{15}$.

Delineate le cause che in pratica resero complicati i rapporti fra l'autorità civile e quella ecclesiastica, resta ancora da vedere in che modo la dottrina cattolica postridentina abbia costruito una teoria delle relazioni Chiesa-Stato; teoria che sarà ripresa e perfezionata dai teorici dello ius publicum ecclesiasticum. Il primo dato da osservare, come rileva Willeart, è l'assoluta mancanza di una teorizzazione delle relazioni fra la Chiesa e gli Stati durante l'Assise tridentina, limitandosi i Padri conciliari a imporre ai sovrani un generico dovere di protezione della Chiesa ${ }^{16}$. I teologi, allora, costruirono le relazioni

\footnotetext{
${ }^{14} I v i$, p. 34.

${ }^{15} \mathrm{Ivi}, \mathrm{p} .33$.

${ }^{16}$ Ivi, p. 533.
} 
della Chiesa con le comunità civili impostandole su un rapporto sinallagmatico: da una parte la Chiesa, infatti, aveva il diritto di vedersi riconoscere una somma di privilegi giudiziari e fiscali ma anche l'obbligo di occuparsi dell'educazione e dell'assistenza delle miserabiles personae; dall'altra, allo Stato correva l'obbligo di eseguire i decreti ecclesiastici concernenti l'eresia e i libri posti all'Indice ${ }^{17}$.

I teologi non si limitarono a delineare una teoria relazionale incentrata su obblighi e diritti reciproci, dove lo Stato fungeva da longa manus della Chiesa circa l'esecuzione di decreti e sentenze giudiziali nel territorio civile e quest'ultima si occupava dell'istruzione e del mantenimento degli indigenti. Essi, infatti, previdero anche un'estensione dell'autorità ecclesiastica (il papato) nel campo della giurisdizione civile. A tal proposito, però, due furono le correnti che si formarono: la teoria della potestas directa Ecclesiae in temporalibus e quella della potestas indirecta Ecclesiae in temporalibus. Secondo la prima concettualizzazione il Papa, detentore del potere delle due spade, poteva destituire il sovrano che si fosse manifestato indegno del potere temporale. Per la teoria del potere indiretto, strutturata in epoca moderna da Francisco de Vitoria, fatta propria da Roberto Bellarmino e Francisco Suárez e ampiamente utilizzata - come si vedrà in seguito - dagli esponenti della scienza giuspubblicistica, il Papa in via diretta non aveva alcuna autorità sulle materie temporali mentre in via eccezionale, per esempio in caso di sovrano eretico, poteva legittimamente intervenire poiché la Chiesa era societas iuridice perfecta e garante di un fine, la salus aeterna animarum, superiore a quello dello Stato ${ }^{18}$.

\section{Il Concilio Vaticano II e le 'relazioni Chiesa-Mondo'}

Il Concilio Ecumenico Vaticano II, celebratosi dall'11 ottobre 1962 all'8 dicembre 1965, ha rappresentato per la Chiesa universale una vera e propria "svolta costituzionale" e ha risposto egregiamente a tutte quelle esigenze di aggiornamento che Papa Roncalli espresse in un

\footnotetext{
${ }^{17}$ Ibidem.

${ }^{18}$ Ivi, pp. 534-535.
} 
celeberrimo discorso, del 25 gennaio 1959, con il quale annunciò l'imminente convocazione dell'Assise conciliare ${ }^{19}$. Per quanto concerne il presente studio, l'attenzione sarà rivolta, in modo particolare, all'autocomprensione della Chiesa così come avvenuta durante il Vaticano II per delineare, poi, gli sviluppi della materia giuspubblicistica fino ai giorni nostri. La convocazione del Concilio si presentò quasi come una "tappa obbligata" poiché la Chiesa appariva scollata dal mondo e le sue istituzioni erano ormai ben lontane da rispondere alle esigenze socio-antropologiche del ventesimo secolo. In più l'ecclesiologia dominante, di matrice neoscolastica, di fatto rimasta inalterata dall'epoca della Controriforma e strutturata in modo piramidale e gerarchica, risultava assolutamente inadeguata e necessitava di essere ripensata alla luce della nuova realtà del Mondo ${ }^{20}$.

L'ecclesiologia presente al momento della convocazione del Concilio, infatti, era quella elaborata, nelle sue linee essenziali, dai Padri conciliari riunitisi a Trento e rispondeva alle esigenze della Chiesa del sedicesimo secolo. Una Chiesa che, attaccata dai riformatori, rispose trincerandosi in una posizione di difesa che se da una parte la salvaguardò, dall'altra l'allontanò dal mondo esterno. L'ecclesiologia post-tridentina, infatti, non teneva in dovuta considerazione il "cambio di passo" intervenuto nella società durante i secoli quindicesimo e sedicesimo. E non mi riferisco alla Riforma, della quale era invece

${ }^{19}$ L'espressione "svolta costituzionale" legata al Concilio Vaticano II è di C. FAnTAPPIÈ, Introduzione storica al diritto canonico, Il Mulino, Bologna 2003, pp. 248-256. Per uno studio sul Concilio Ecumenico Vaticano II si veda, ex multis, G. Lo Castro, La qualificazione giuridica delle deliberazioni conciliari nelle font $i$ di diritto canonico, Giuffrè, Milano 1970; G. Alberigo (ed.), Decisioni dei Concili ecumenici, UTET, Torino 1978; G. Alberigo (ed.), Il Vaticano II fra attese e celebrazioni, Il Mulino, Bologna 1995; H. Jedin, Breve storia dei concili, Morcelliana, Brescia 2006; L. F. Capovilla, Ricordi del Concilio. Siamo appena all'aurora, La Scuola, Brescia 2011; G. Dossetti, Il Vaticano II. Frammenti di una riflessione, a cura di F. Margiotta Broglio, Il Mulino, Bologna 2012; PH. Chenaux, Il Concilio Vaticano II, Carocci, Roma 2012; G. Colombo, Il Concilio Vaticano II. Discorsi e scritti, a cura di I. Biffi, Jaca Book, Milano 2013.

${ }^{20}$ Cfr. J. Estrada, La Iglesia: identidad y cambio. El concepto de Iglesia del Vaticano I a nuestros dias, Ediciones Cristianidad, Madrid 1985, pp. 28-52. 
ben consapevole, ma ad un dato ancora più rilevante: era ormai finita l'epoca della christianitas, intesa come unica Respublica christiana di stampo europeo, ed incalzava rapidissimamente, al contrario, una società pluralistica e fortemente secolarizzata in cui la disaffezione nei confronti delle istituzioni ecclesiastiche e, più in generale, nei confronti del sentimento religioso, si faceva sempre più evidente ${ }^{21}$. Appare degno di sottolineatura ancora un aspetto. Nella preparazione e nello sviluppo del Concilio ci furono, però, posizioni teologiche contrastanti fra i Padri e tutta la rilettura che la Chiesa voleva fare di se stessa in relazione al mondo contemporaneo, incontrava la sua prima difficoltà in questa differenze di visioni ecclesiologiche. Da una parte, visione del mondo come luogo dal quale difendersi oppure come luogo con il quale era necessario relazionarsi; dall'altra, visione della Chiesa come ordine giuridico fisso e inamovibile oppure come realtà sacramentale e mutevole ${ }^{22}$.

Delineato lo status quo ante, è ora possibile prendere in considerazione i documenti conciliari che maggiormente si sono occupati della autocomprensione della Chiesa e che hanno determinato, conseguentemente, un cambiamento sostanziale nelle relazioni con il Mondo. Faccio riferimento alla Costituzione dogmatica Lumen Gentium ${ }^{23}$ e alla Costituzione pastorale Gaudium et Spes ${ }^{24}$. Entrambi i documenti, infatti, si completano a vicenda e ciascuno, offrendo rispettivamente una "nuova visione di Chiesa" e una "nuova modalità di relazione con il mondo esterno", costituisce il contenitore essenziale per lo sviluppo della scienza giuspubblicistica nell'epoca postconciliare. Proprio perché ciascun documento offre una parte essenziale per la costruzione del "nuovo" ius publicum ecclesiasticum, mi occuperò in questo paragrafo del "nuovo" modo in cui la Chiesa si è autodefinita

\footnotetext{
${ }^{21}$ P. Grossi, L’Europa del diritto, pp. 83-87.

${ }^{22}$ Cfr. J. Estrada, op. cit., pp. 27-37.

${ }^{23}$ Concilium Oecumenicum Vaticanum II, Constitutio dogmatica de Ecclesia Lumen Gentium (21 Novembris 1964), in: Enchiridion Vaticanum I, nn. 284-445.

${ }^{24}$ Concilium Oecumenicum Vaticanum II, Constitutio pastoralis de Ecclesia in mundo huius temporis Gaudium et Spes (7 Decembris 1965), in: Enchiridion Vaticanum I, nn. 1319-1644.
} 
per poi trattare, in quello seguente, delle relazioni della stessa con il Mondo.

\subsection{La Costituzione dogmatica sulla Chiesa Lumen Gentium}

Nella Costituzione dogmatica Lumen Gentium è descritta l'essenza della Chiesa con espressioni che manifestano chiaramente il nuovo approccio emergente dal Concilio. Così, per esempio, la denominazione della Chiesa come mistero (LG 1) supera la concezione della stessa come realtà prevalentemente visibile e socio-giuridica, accentuando, al tempo stesso, il suo aspetto invisibile in analogia con la natura umana e divina di Gesù Cristo $(\mathrm{LG} 8)^{25}$. Il nucleo della Chiesa, allora, non s'individua nelle istituzioni ma in un'azione trinitaria dove risulta prevalente l'aspetto pneumatologico (LG 7 $)^{26}$.

Particolarmente interessante, per il tema del presente lavoro e in prospettiva di verificare le nuove modalità dei rapporti fra la Chiesa $\mathrm{e}$ il mondo esterno, è la relazione che il documento conciliare stabilisce fra la Chiesa e il Regno anche se, come rileva Estrada, il Concilio non ha inteso precisare teologicamente il modo in cui si relazionano le due entità ${ }^{27}$. Pur affermandosi chiaramente che la Chiesa è il Regno di Cristo (LG 3), tuttavia si chiarisce che il Regno non è di questo mondo e il popolo di Dio lo introduce nella storia (LG 13). Inoltre, non sono neppure precisate le relazioni fra il Regno di Cristo e di Dio oppure se si tratti di realtà simili o differenti. Si afferma che Gesù ha dato inizio alla Chiesa annunciando il Regno e che la Chiesa abbia ricevuto la missione di instaurarlo in tutte le genti costituendone in terra il germe e l'inizio (LG 5); per continuare, poco dopo, asserendo che il fine della Chiesa è di estendere il Regno di Dio a tutta la terra entrando nella storia degli uomini ma trascendendo i tempi e i confini

\footnotetext{
${ }^{25}$ Cfr. K. Rahner - H. Vorgrimler (edd.), I documenti del Concilio Vaticano II, 7 ed., Edizioni Paoline, Roma 1968, pp. 112-114; J. Estrada, op. cit., pp. 59-62; 68-70.

${ }^{26}$ Cfr. K. Rahner - H. Vorgrimler (edd.), op. cit,, p. 113; J. Estrada, op. cit., p. 67.

${ }^{27}$ J. EstradA, op. cit., p. 67.
} 
dei popoli (LG 9) ${ }^{28}$. Il documento in esame, quindi, sottolineando che le due entità salvifiche non sono equiparabili ma al tempo stesso intimamente vincolate, evidenzia la dimensione missionaria ed escatologica della Chiesa (6-8) che, pellegrina nella storia, si presenta al mondo come sacramento universale (LG 48-49) e come germe di unità e di speranza (LG 14-17) $)^{29}$.

\subsection{La Costituzione pastorale Gaudium et Spes}

Il Concilio Vaticano II, presentando con la Costituzione dogmatica Lumen Gentium un'ecclesiologia di "comunione" ben lontana da quella "gerarchica" della Controriforma, ha determinato, a sua volta, un naturale ripensamento dei rapporti fra la Chiesa e le comunità politiche mediante la Costituzione pastorale Gaudium et Spes. Se, infatti con la Costituzione dogmatica Lumen Gentium si è potuto comprendere il ruolo, la missione e la definizione stessa di Chiesa "nel" Mondo, con la Gaudium et Spes si mette in relazione la Chiesa "con" il Mondo. Secondo la riflessione di Karl Rahner, due sono le principali caratteristiche che si riscontrano in questo documento. Innanzitutto una risposta positiva della Chiesa ad un mondo - anche laico e laicista - che in trepidante attesa si chiedeva se essa avrebbe saputo dichiararsi solidale con l'umanità e tenuto in debita considerazione i problemi che la affliggevano ${ }^{30}$. In secondo luogo, il documento in analisi ha il "merito" - il "coraggio", secondo le parole del teologo gesuita di Friburgo - di non dare definizioni teologiche o di principio ma, viceversa, offrire direttive concrete per l'uomo contemporaneo; direttive che sono indirizzate ai membri della Chiesa ma, vista la loro applicabilità all'umanità in generale, anche dirette, sotto forma di inviti e raccomandazioni, a tutti gli uomini ${ }^{31}$.

Per comprendere esattamente la portata innovatrice del documento, mi sembra doveroso spendere ancora qualche parola sul

\footnotetext{
${ }^{28}$ Ibidem. Cfr. K. RAhner - H. Vorgrimler (edd.), op. cit,, p. 115.

${ }^{29}$ J. EstradA, op. cit., pp. 70-75.

${ }^{30}$ Cfr. K. Rahner - H. Vorgrimler (edd.), op. cit,, pp. 266-267.

${ }^{31}$ Ivi, p. 268.
} 
Proemio che in appena tre numeri offre le coordinate necessarie per comprendere il profondo mutamento d'atteggiamento della Chiesa non più preoccupata di difendersi dai doctores protestantium - nei rapporti con il mondo civile. Sin dalle prime parole, infatti, si comprende come la Chiesa intenda unirsi all'intera famiglia umana, ponendosi in un rapporto intimamente solidale con il genere umano e la sua storia (GS 1). Parlando di intera famiglia umana, il Concilio intende riferirsi, come ho già accennato, non solo ai figli della Chiesa o a coloro che invocano il nome di Cristo ma, indistintamente, a tutti gli uomini affinché conoscano il modo in cui intende configurare la presenza della Chiesa nel Mondo (GS 2). Tale proposito, sovvertendo gran parte degli schemi gerarchico-istituzionali dell'ecclesiologia post-tridentina, prenderà la forma del "dialogo", inteso come strumento mediante il quale la Chiesa può offrire all'umanità globalmente intesa «le energie di salvezza che, sotto la guida dello Spirito Santo, riceve dal suo Fondatore» $(\mathrm{GS} 3)^{32}$.

Caliamoci, adesso, nello specifico e proviamo ad evidenziare le linee direttrici che il documento conciliare ci offre per la regolazione dei rapporti "Chiesa-Mondo". Esse si trovano all'interno del capitolo che tratta della vita della comunità politica e, più precisamente, al numero settantasei dal titolo Communitas politica et Ecclesia ${ }^{33}$.

${ }^{32}$ Cfr. K. Rahner - H. Vorgrimler (edd.), op. cit,, p. 269; J. Estrada, op. cit., pp. 94-95.

${ }^{33}$ Concilium Oecumenicum Vaticanum II, Constitutio pastoralis Gaudium et Spes, caput IV, De vita communitatis politicae, n. 76, Communitas politica et Ecclesia, in: Enchiridion Vaticanum I, nn. 1579-1584: «Magni momenti est, praesertim ubi societas pluralistica viget, ut rectus respectus relationis inter communitatem politicam et Ecclesiam habeatur, utque inter ea, quae christifideles, sive singuli sive consociati, suo nomine tamquam cives, christiana conscientia ducti, et ea, quae nomine Ecclesiae una cum pastoribus suis agunt, clare distinguatur. Ecclesia, quae, ratione sui muneris et competentiae, nullo modo cum communitate politica confunditur, neque ad ullum, systema politicum alligatur, simul signum est et tutamentum transcendentiae humanae personae. Communitas politica et Ecclesia in proprio campo ab invicem sunt independentes et autonomae. Ambae autem, licet diverso titulo, eorumdem hominum vocationi personali et sociali inserviunt. Quod servitium eo efficacius in omnium bonum exercebunt, quo ambae melius sanam cooperationem inter se colunt, 
Analizzando questa parte di Costituzione pastorale appena richiamata, è possibile rilevare fin da subito un elemento di discontinuità rispetto al panorama storico-culturale nel quale nasce e si sviluppa lo ius publicum ecclesiasticum. Nel documento conciliare, infatti, la comunità politica e la Chiesa sono inserite all'interno di una «societas pluralistica» ${ }^{34}$. Ciò premesso, si afferma la necessità che la Chiesa e la comunità politica, all'interno di questo contesto pluralistico, maturino una chiara consapevolezza sul modo di regolare i loro rapporti. Subito dopo si precisa che, anche se in un rapporto di collaborazione reciproca, la Chiesa non deve essere confusa - «ratione sui muneris et competentiae» ${ }^{35}$ - con la comunità politica poiché essa non è legata ad

attentis quoque locorum temporumque adiunctis. Homo enim ad solum ordinem temporalem non coarctatur, sed in historia humana vivens aeternam suam vocationem integre servat. Ecclesia vero, in Redemptoris amore fundata, ad hoc confert ut intra nationis terminos et inter nationes iustitia et caritas latius vigeant. Evangelicam veritatem praedicando atque omnes navitatis humanae provincias, per suam doctrinam et testimonium a christifidelibus exhibitum, illuminando, etiam politicam civium libertatem et responsabilitatem reveretur atque promovet. Apostoli ipsorumque successores et horum cooperatores, cum mittantur ut hominibus Christum mundi Salvatorem annuntient, in suo apostolatu exercendo Dei potentia innituntur, qui persaepe in testium infirmitate virtutem Evangelii manifestat. Quicumque enim Dei verbi ministerio se devovent, utantur oportet viis et subsidiis Evangelio propriis, quae in pluribus a terrenae civitatis subsidiis differunt. Res quidem terrenae et ea, quae in hominum condicione hunc mundum exsuperant, arcte inter se iunguntur, et ipsa Ecclesia rebus temporalibus utitur quantum propria eius missio id postulat. Spem vero suam in privilegiis ab auctoritate civili oblatis non reponit; immo quorundam iurium legitime acquisitorum exercitio renuntiabit, ubi constiterit eorum usu sinceritatem sui testimonii vocari in dubium aut novas vitae condiciones aliam exigere ordinationem. Semper autem et ubique ei fas sit cum vera libertate fidem praedicare, socialem suam doctrinam docere, munus suum inter homines expedite exercere necnon iudicium morale ferre, etiam de rebus quae ordinem politicum respiciunt, quando personae iura fundamentalia aut animarum salus id exigant, omnia et sola subsidia adhibendo, quae Evangelio et omnium bono secundum temporum et condicionum diversitatem congruant. Fideliter Evangelio adhaerens et suam missionem in mundo exercens, Ecclesia, cuius est, quidquid verum, bonum et pulchrum in communitate humana invenitur, fovere ac elevare, pacem inter homines ad Dei gloriam roborat».

${ }^{34}$ Ibidem.

${ }^{35}$ Ibidem. 
alcun sistema politico. Da ciò discende una "dichiarazione di principio" dalla portata notevolissima: «communitas politica et Ecclesia in proprio campo ab invicem sunt indipendentes et autonomae» ${ }^{36}$. L'autonomia e l'indipendenza sono i tratti fondamentali di queste due realtà che agiscono, con mezzi diversi ma egualmente efficaci, a sevizio della vocazione personale e sociale delle stesse persone umane.

Un altro elemento centrale, oltre al panorama pluralistico della società, è quello della sana cooperazione (sana cooperatio) ${ }^{37}$ che dovrebbe sussistere per un'azione efficace della Chiesa e della comunità politica nel perseguire lo stesso bene comune. Infatti, il compito di entrambe - il servizio verso la vocazione personale e sociale delle stesse persone umane - si rafforza in misura direttamente proporzionale all'esistenza di una sana collaborazione tra le due realtà e nel pieno rispetto dei diversi modi utilizzabili secondo le circostanze di luogo e di tempo.

Infine, è possibile evidenziare come il documento conciliare abbia voluto inscindibilmente collegare il concetto di sana cooperatio a quello di persona umana che, come indicato mirabilmente dal Doctor Angelicus ${ }^{38}$, è «principio, soggetto e fine di tutte le istituzioni sociali» ${ }^{39}$. In questa visione di aiuto reciproco la Chiesa, fondata ed intrisa dell'amore del suo Divino Fondatore, aiuta le Nazioni a comprendere e ad attuare la giustizia in chiave antropologica nonché a rispettare e promuovere la libertà politica e la responsabilità dei

\footnotetext{
${ }^{36}$ Ibidem.

${ }^{37}$ Ibidem.

${ }^{38}$ Thomas Aquinatis, I Ethic., Lect. 1, in: Opera Omnia, XXI, apud Anissonios, J. Postel et Cl. Rigaud, Lugduni 1660-1685, p. 2, col. 1: «Sciendum est autem, quod quia homo naturaliter est animal sociale, utpote qui indiget ad suam vitam multis, quae sibi ipse solus praeparare non potest; consequens est, quod homo naturaliter sit pars alicuis multitudinis, per quam praestetur sibi auxilium ad bene vivendum».

${ }^{39}$ Concilium Oecumenicum Vaticanum II, Constitutio pastoralis Gaudium et Spes, n. 1396: "principium, subiectum et finis omnium institutorum socialium est et esse debet humana persona, quippe quae, suapte natura, vita sociali omnino indigeat».
} 
cittadini ${ }^{40}$. A dimostrazione di una riconosciuta importanza dell'aiuto reciproco che è bene sussista fra la Chiesa e la comunità politica nella gestione dei loro rapporti, si afferma che le res terrenae, insieme a quelle «hunc mundum exsuperant» ${ }^{41}$, sono strettamente unite e che la stessa Chiesa le utilizza nella misura in cui ciò sia utile alla propria missione ${ }^{42}$. Naturalmente, la possibilità che la Chiesa si serva di queste cose terrene è commisurata alla consapevolezza che essa non deve agire esclusivamente per utilizzare i beni che le vengono offerti dall'autorità civile. Anzi, la stessa Chiesa non dovrà esercitare determinati diritti, seppur legittimamente acquisiti, qualora il loro esercizio possa far dubitare della sincerità della sua testimonianza ${ }^{43}$. Sono fermamente convinto nel ritenere, come sostiene Rahner, che tale "dichiarazione di rinuncia" sia uno dei vertici più importanti del Concilio perché serve a cambiare totalmente l'immagine della presenza della Chiesa nel mondo; una presenza che la porterà ad utilizzare i beni materiali solo quando essi siano un mezzo per il raggiungimento del suo fine ultimo e non solo perché costituiscono, anche per via concordataria, un diritto quesito ${ }^{44}$.

\subsection{La Dichiarazione sulla libertà religiosa Dignitatis humanae}

Un'ultima notazione riguarda il richiamo a un altro documento conciliare che deve essere letto sinotticamente al numero settantasei

${ }^{40}$ Ivi, n. 1581: «Ecclesia vero, in Redemptoris amore fundata, ad hoc confert ut intra nationis termino set inter nationes iustitia et caritas latius vigeant. Evangelicam veritatem praedicando atque omnes navitatis humanae provincias, per suam doctrinam et testimonium a christifidelibus exhibitum, illuminando, etiam politicam civium libertatem et responsabilitatem reveretur atque promovet».

${ }^{41}$ Ivi, n. 1583.

${ }^{42}$ Ibidem: «Res quidem terrenae e tea, quae in hominum condicione hunc mundum exsuperant, arcte inter se iunguntur, et ipsa Ecclesia rebus temporali bus utitur quantum propria eius missio id postulat».

${ }^{43}$ Ibidem: «Spem vero, suam in privilegiis ab auctoritate civili oblatis non reponit; immo quorundam iurium legitime acquisito rum exercitio renuntiabit, ubi constiterit eorum usu sinceritatem sui testimonii vocari in dubium aut novas vitae condiciones aliam exigere ordinationem».

${ }^{44}$ Cfr. K. Rahner - H. Vorgrimler (edd.), op. cit,, p. 295. 
della Costituzione pastorale Gaudium et Spes per avere il quadro completo della nuova impostazione teorica delle relazioni "Chiesa-Mondo”. Si tratta della Dichiarazione sulla libertà religiosa Dignitatis humanae del 7 dicembre 1965, in particolar modo il numero tredici. Prima di vederne il contenuto e le implicazioni per il tema di questa indagine, è opportuno precisare che la Dichiarazione non si occupa in generale della libertà religiosa nella Chiesa ma, più correttamente, delle relazioni delle persone fisiche e morali nella società politica e umana e del loro rapporto con il potere pubblico in tema di religione $(\mathrm{DH} 2)^{45}$. Al numero tredici del documento conciliare si afferma che la libertas Ecclesiae è il principio fondamentale nella regolazione dei rapporti fra la Chiesa e le comunità politiche ${ }^{46}$. Inoltre, si statuisce una sostanziale concordia fra la libertas Ecclesiae e la libertas religiosa,

\footnotetext{
${ }^{45}$ Ivi, p. 844.
}

${ }^{46}$ Concilium Oecumenicum Vaticanum II, Declaratio de libertate religiosa Dignitatis Humanae (7 Decembris 1965), II, Libertas religiosa sub luce Revelationis, n. 13, Libertas Ecclesiae, in: Enchiridion Vaticanum I, nn. 1075-1077: «Inter ea quae ad bonum Ecclesiae, immo ad bonum ipsius terrenae civitatis spectant et ubique semperque servanda sunt atque ab omni iniuriadefendenda, illud certe praestantissimum est, ut Ecclesia tanta perfruatur agendi libertate, quantam salus hominum curanda requirat. Haec enim libertas sacra est, qua Unigenitus Dei Filius ditavit Ecclesiam acquisitam sanguine suo. Ecclesiae sane adeo propria est, ut qui eam impugnant, iidem contra Dei voluntatem agant. Libertas Ecclesiae est principium fundamentale in relationibus inter Ecclesiam et potestates publicas totumque ordinem civilem. In societate humana et coram quavis potestate publica Ecclesia sibi vindicat libertatem, utpote auctoritas spiritualis, a Christo Domino constituta, cui ex divino mandato incumbit officium eundi in mundum universum et Evangelium praedicandi omni creaturae. Libertatem pariter sibi vindicat Ecclesia prout est etiam societas hominum qui iure gaudent vivendi in societate civili secundum fidei christianae praescripta. Iamvero si viget ratio libertatis religiosae non solum verbis proclamata neque solum legibus sancita, sed etiam cum sinceritate in praxim deducta, tunc demum Ecclesia stabilem obtinet et iuris et facti condicionem ad necessariam in missione divina exsequenda independentiam, quam auctoritates ecclesiasticae in societate presse pressiusque vindicarunt. Simulque Christifideles, sicut et ceteri homines, iure civili gaudent ne impediantur in vita sua iuxta conscientiam agenda. Concordia igitur viget inter libertatem Ecclesiae et libertatem illam religiosam, quae omnibus hominibus et communitatibus est tamquam ius agnoscenda et in ordinatione iuridica sancienda». 
che deve essere riconosciuta come diritto a tutti gli esseri umani e garantita dall'ordinamento giuridico delle società civili ${ }^{47}$.

Sulla "concordia" fra i concetti "libertas Ecclesiae" e "libertas religiosa", la dottrina si è espressa in termini diversi. Da una parte, alcuni studiosi hanno ravvisato una sostanziale identità fra la libertà della Chiesa e la libertà religiosa osservando che la prima rientra nella seconda, con il risultato che lo ius libertatis della Chiesa verrebbe a costituire una determinazione dello ius ad libertatem religiosam ${ }^{48}$. Dall'altra, in senso opposto, si sono espressi canonisti che hanno sottolineato come il Concilio abbia voluto tenere ben distinti i due concetti proprio sulla base dello stesso numero tredici della Dichiarazione Dignitatis Humanae nella parte in cui afferma la sussistenza

${ }^{47}$ Ibidem.

${ }^{48}$ Cfr. L. Musselli, Chiesa cattolica e Comunità politica, Cedam, Padova 1975, p. 73. L'Autore rafforza l'idea della compenetrazione fra libertà religiosa e libertà della Chiesa quando sostiene che «la libertà della Chiesa è rispettata colà ove sia rispettata la libertà religiosa e che i cristiani come gli altri uomini godono del diritto civile di non essere impediti di vivere secondo la propria coscienza. Possiamo notare come l'unica potestas che la Chiesa rivendica verso la civitas sia una potestas libertatis e cioè la pretesa al riconoscimento di un diritto soggettivo ed originario della Chiesa, come realtà unitaria e nello stesso tempo sintetica di tutte le comunità ecclesiali locali, alla libertà, diritto che, per l'ambito in cui si pone e per le modalità con cui è proclamato, si può qualificare come peculiare espressione e specificazione del generale diritto di libertà religiosa, inteso nel suo senso più lato di libertà di manifestazione interiore del proprio credo sia nei rapporti con il pubblico potere, che nei rapporti con gli altri consociati e coi gruppi in cui questi si raccolgono» (Ivi, pp. 74; 76). Secondo questa impostazione "coordinazionista", allora, "venendo la libertas Ecclesiae, di cui si parla al c. 13 della Dignitatis Humanae come criterio generale dei rapporti Stato-Chiesa, a coincidere dal punto di vista contenutistico col diritto di libertà religiosa, si può dire che, come il principio della libertà della Chiesa è il cardine dei rapporti tra la Chiesa cattolica e lo Stato, così l'istituto della libertà religiosa, quale configurato dal Concilio, è lo strumento generale di coordinazione tra la società civile ed il fenomeno religioso; infatti a nient'altro è finalizzata la dichiarazione Dignitatis Humanae che non sia questo semplicissimo postulato: che i singoli e le comunità confessionali (tra le quali in primis la Chiesa cattolica) possano godere nell'ambito della società politica dei loro inalienabili diritti di libertà religiosa» (Ivi, p. 77). 
di una concordia fra la libertà della Chiesa e la libertà religiosa. Il termine "concordia", infatti, non solo non può essere confuso con quello di "identità" ma, addirittura, può sussistere solo tra realtà distinte ${ }^{49}$. A parte le diverse posizioni della dottrina, quel che preme rilevare è che questa Dichiarazione conciliare, insieme alle due Costituzioni prima esaminate, offre un insieme di principi e di concetti di basilare importanza per disegnare le strutture e i contenuti dei rapporti fra la Chiesa e le comunità politiche $\mathrm{e}^{50}$.

${ }^{49}$ Cfr. G. Dalla Torre, La città sul monte. Contributo ad una teoria canonistica sulle relazioni fra Chiesa e Comunità politica, 3 ed., editrice a.v.e., Roma 2007, p. 116. Per motivare la differenza fra libertas Ecclesiae e libertà religiosa, Dalla Torre chiarisce che «la libertà religiosa è, nell'accezione conciliare, un concetto negativo, concretizzandosi nell'immunità da coercizioni esterne in materia religiosa o di coscienza. La libertas Ecclesiae, al contrario è un concetto positivo, per il quale la Chiesa nel suo agire deve godere di «tanta libertà quanto le è necessaria per provvedere alla salvezza degli esseri umani» (dich. Dignitatis humanae \$13). Il primo concetto attiene all'ordine interno dello Stato; l'altro, al contrario, riguarda l'ordine esterno» (Ivi, p. 116). Ma la distinzione più evidente fra la libertà religiosa e la libertas Ecclesiae, secondo Dalla Torre, si coglie guardando alla loro intima natura. Ed infatti «la prima si pone sul piano della creazione: è quindi un diritto naturale che, come tale, spetta a ciascun uomo che viene a questo mondo. La stessa denominazione della dichiarazione conciliare Dignitatis humanae, sta a indicare che il diritto a non essere forzato ad agire contro la propria coscienza né impedito ad agire in conformità ad essa non nasce dalla volontà di un legislatore positivo, ma si radica nella stessa dignità dell'essere umano. La seconda, al contrario, si pone sul piano della redenzione: è un diritto divino positivo, che spetta solo alla Chiesa nella misura in cui i suoi contenuti sono qualificati dall'essere stesso della istituzione ecclesiastica e dalla missione che il suo Fondatore ha affidato ad essa, e solo ad essa, di continuare la sua opera nel tempo, fino alla consumazione della storia» (Ivi, p. 117).

${ }^{50}$ Sul concetto di 'libertas Ecclesiae' e 'libertà religiosa' come elementi di individuazione di una teoria di relazioni fra Chiesa e Comunità politica si veda, in modo particolare, G. LAJOLO, «Libertas Ecclesiae»: principio fondamentale nelle relazioni tra Chiesa e Stato, La scuola cattolica 5(1970), pp. 3-31;113-134; L. SPINELLI, Libertas ecclesiae. Lezioni di diritto canonico, Giuffrè, Milano 1979; L. Mistò, "Libertas religiosa» «libertas Ecclesiae». Il fondamento della relazione Chiesa-comunità politica nel quadro del dibattito postconciliare in Italia, Morcelliana, Brescia 1982. 
Dall'analisi di questi tre documenti, ciascuno di vitale importanza, credo sia possibile affermare con sicurezza che il Concilio Vaticano II ha determinato un ripensamento così forte delle relazioni "Chiesa-Mondo" da poter parlare di un vero e proprio "dissesto strutturale" dal punto di vista della scienza giuspubblicistica. Se, infatti, le relazioni fra queste due entità sono ora impostate sulla sana cooperatio, ne consegue che il diritto pubblico ecclesiastico perderà il suo afflato apologetico e al tempo stesso, non dovendo più affaticarsi a dimostrare necessariamente la perfezione giuridica della Chiesa e il suo essere ordinamento giuridico primario, acquisirà una sempre maggiore consapevolezza della sua importanza nella gestione dei rapporti fra la Chiesa e le comunità politiche a partire da una nuova autodeterminazione di apertura verso il mondo esterno ${ }^{51}$. Ed è una nuova autodeterminazione perché il nodo centrale non sarà più la "difesa" ma, come ci ha ricordato più volte Papa Francesco, la "creazione di ponti" in grado di cementare i rapporti fra persone, istituzioni, comunità politiche e religiose.

\section{Historical and juridical considerations about the 'connections Church-World' in the Council of Trent and in the Second Vatican Council}

The present work is a conference in the Faculty of Law of the Catholic University of Murcia (Spain) and want to consider the importance of the

\footnotetext{
${ }^{51}$ Quando si fa riferimento all'apertura del Concilio Vaticano II, vogliamo mettere in evidenza che la nuova 'auto-determinazione' della scienza giuspubblicistica è soltanto la conseguenza di uno dei molti principi che - anche se in maturazione ormai già da molto tempo - si sono concretizzati solo con questo Concilio. Facciamo riferimento, ad esempio, alla valorizzazione del Popolo di Dio e alla sua attiva partecipazione all'esercizio dei tria munera Christi; alla valorizzazione della collegialità episcopale, a discapito di una centralizzazione del potere nella persona del Pontefice e della Curia Romana; all'affermazione del principio dell'Ecumenismo e della Evangelizzazione dei Popoli. Cfr. L. Musselli, Storia del diritto canonico. Introduzione alla storia del diritto e delle istituzioni ecclesiali, Giappichelli, Torino 2007, pp. 101-106.
} 
juridical science named ius publicum ecclesiasticum and the connections between the Church and the Civil Governments. The focus of the study, in my intention, is to underline the important 'juridical culture' of this special part of the Canon law.

Parole Chiave: 'relazioni Chiesa-Mondo'; Concilio di Trento; Concilio Vaticano II; Costituzione dogmatica sulla Chiesa Lumen Gentium; Costituzione pastorale sulla Chiesa nel mondo contemporaneo Gaudium et Spes; Dichiarazione sulla libertà religiosa Dignitatis Humanae.

Keywords: 'connections Church-World'; Council of Trent; Second Vatican Council; Dogmatic constitution on the Church Lumen Gentium; Pastoral constitution on the Church in the modern world Gaudium et Spes; Declaration on religious freedom Dignitatis humanae.

Nota o Autorze:

Prof. Matteo Nacci - is full professor of History of Law and director of Department of History of Law in the Pontifical Lateran University. 